\title{
Depressão pós-parto na adolescência: um problema relevante?
}

\section{Is postpartum depression in adolescents an issue of concern?}

Recife, 12 de setembro de 2006

Prezado editor,

$\mathrm{O}$ artigo de Moraes et $\mathrm{al}^{3}$ (2006) sobre depressão pós-parto (DPP), publicado na Revista de Saúde Pública, é de um valor irrefutável, principalmente por ser o primeiro de base populacional realizado no País que analisou uma amostra numericamente tão significativa $(\mathrm{n}=401)$. No entanto, algumas considerações metodológicas merecem ser discutidas. A escala de avaliação utilizada para o diagnóstico de DPP, a Hamilton (HAM-D), não foi apropriada. A HAM-D é um instrumento diagnóstico amplamente utilizado na psiquiatria, mas foi elaborada para avaliar e quantificar a sintomatologia depressiva em portadores de transtornos do humor. Não é recomendada para outro tipo de paciente, nem para pessoas normais e não constitui instrumento diagnóstico para identificação de depressão. ${ }^{4}$

Escalas mais apropriadas já são disponíveis no Brasil, validadas e específicas para o diagnóstico da DPP, a exemplo da Edimburgh Postnatal Depression Scale $^{5}$ (EPDC) e da Postpartum Depression Screening Scale $^{2}$ (PDSS), citadas pelos autores. As escalas utilizadas para depressão geral têm limitações importantes quanto à capacidade de detectar sintomas depressivos em mulheres no pós-parto. Um dos pontos de debate envolvendo o uso desses instrumentos é a inclusão de sintomas que são considerados reações fisiológicas normais do puerpério, como fadiga e distúrbios do sono, e a exclusão de outros importantes para o diagnóstico da DPP (como sentimentos ambivalentes em relação à criança e de opressão pela responsabilidade de cuidar, dificuldade em revelar seus sintomas por receio de serem estigmatizadas, entre outros).
Não há trabalhos no Brasil que avaliem a DPP na adolescência. Moraes et $\mathrm{al}^{3}$ afirmam que as precárias condições socioeconômicas e a não aceitação da gravidez são os fatores que mais influenciam o aparecimento da depressão no puerpério. Considerando a gravidez na adolescência como um problema de caráter social, partindo do pressuposto de que a gravidez nesta fase é mais freqüente nas classes menos favorecidas e encarada, na maioria das vezes, como inoportuna, com conseqüências biológicas, psicológicas e sociais negativas, é relevante se investigar se as adolescentes estão mais predispostas a apresentar DPP. Entretanto, é importante frisar que, apesar de a maioria dos estudos apontar o fato de que a gravidez na adolescência ocorre sem o planejamento prévio, numa etapa de aprendizagem da sexualidade, como fruto de relacionamentos afetivos instáveis, nem sempre ela é indesejada. Muitos rapazes associam a paternidade a uma melhoria do status social, por meio da qual passam a ser mais respeitados pela sua masculinidade; e, as moças associam a maternidade ao seu "projeto" de realização pessoal. ${ }^{1}$

No mestrado em Hebiatria da Universidade de Pernambuco está sendo realizada a coleta de dados de uma dissertação cujo objetivo é avaliar a ocorrência de DPP em puérperas adolescentes da rede pública de saúde da cidade do Recife, PE. Foram avaliadas, até o momento, 50 adolescentes entre o segundo e o sexto mês do puerpério, tendo sido encontrada a prevalência de $30 \%$ (por meio da PDSS). A idade média foi 17,4 anos (14 a 19 anos).

Moraes et $\mathrm{al}^{3}$ (2006) alertam para a relevância da DPP como problema de saúde pública e enfatizam a necessidade de estratégias de prevenção e tratamento. As puérperas são examinadas por seus obstetras ou clínicos gerais em consultas focadas na recuperação física após o parto e, quando apresentam de- 
pressão, muitas vezes não são adequadamente diagnosticadas. O subdiagnóstico da DPP é muito mais grave quando ocorre na adolescente, por ser a gravidez na adolescência de alto risco tanto do ponto de vista obstétrico, quanto neonatal e alvo de peculiaridades.

Assim sendo, propomos a PDSS como escala de triagem a ser utilizada por profissionais de saúde da rede básica de assistência ao adolescente. É uma escala facilmente aplicável e fornece condições para que os profissionais de saúde que prestam assistência ao adolescente tornem-se capazes de identificar, intervir e se, necessário, encaminhar os casos de depressão pós-parto em adolescentes

\section{REFERÊNCIAS}

1. Barbosa EMS, Silva KVP, Petribu K, Frota MLM, Amorim VCSA. Comportamento sexual e reprodutivo de adolescentes brasileiros: análise das variáveis adotadas nas pesquisas científicas. Rev Bras Saúde Materno-Infantil. [No prelo]

2. Cantilino A. Tradução para o português e estudo de validação da Postpartum Depression Scale na população brasileira [dissertação de mestrado] Recife: Universidade Federal de Pernambuco; 2003.

3. Moraes IGS, Pinheiro RT, Silva RA, Horta BL, Sousa $P L R$, Faria AD. Prevalência da depressão pós-parto e fatores associados. Rev Saúde Pública. 2006;40:65-70.

\section{Resposta do autor}

Pelotas, 19 de setembro de 2006

Prezado editor,

Gostaríamos, em primeiro momento, de agradecer a avaliação positiva feita ao nosso trabalho ${ }^{8}$ pela equipe da Universidade de Pernambuco em Carta ao Editor envidada à esta prestigiosa revista.

Especificamente em relação à questão levantada na mesma carta, sobre a escolha da escala de avaliação para a assistência de profissionais de saúde mental.

Edilene Maria da Silva Barbosa

Mariana da Cruz Silva*

Michele Ribeiro da Silva*

Faculdade de Enfermagem Nossa Senhora das Graças

Universidade de Pernambuco. Recife, PE

Maria Cristina Montenegro*

Kátia Petribú

Faculdade de Ciências Médicas

Universidade de Pernambuco. Recife, PE

4. Moreno RA, Moreno DH. Escalas de avaliação clínica em psiquiatria e psicofarmacologia: escalas de avaliação para depressão de Hamilton (HAM-D) e Montgomery-Asberg (MADRS). Rev Psiquiatr Clín. $1998 ; 25(5): 1-17$.

5. Santos MF, Martins FC, Pasquali L. Escala de autoregistro de depressão pós-parto: estudo no Brasil. In: Gorenstein C, Andrade LH, Zuardi AW, editores. Escalas de avaliação clínica em psiquiatria e psicofarmacologia. São Paulo: Lemos Editorial; 2000. p. 97-101.

de depressão utilizada no estudo, existem dois pontos que devem ser destacados: 1) a PDSS ainda não havia sido validada durante a realização do trabalho (que aconteceu em 2000 e a validação ${ }^{3}$ só foi feita em 2003); e 2) a EPDS, no momento do trabalho, contava apenas com uma validação ${ }^{6}$ recente, em uma população de classe média.

A respeito das limitações da Hamilton Depression Scale (HAM-D), julgamos pertinente salientar que se trata de uma escala desenvolvida há mais de 40 anos que ainda mantém sua posição de escala administrada por pesquisadores mais usada mundialmente. ${ }^{5} \mathrm{~A}$ sua escolha para a investigação aconteceu pela ampla utilização em pesquisa para o estabelecimento de 
critérios clínicos de inclusão, medidas de evolução ou de recuperação de um episódio depressivo. ${ }^{7}$ Além disso, o fato de a HAM-D ser aplicada por um avaliador treinado permite uma avaliação mais acurada do quadro depressivo. De fato, como sugere a carta enviada, a inclusão de sintomas somáticos na escala poderia aumentar a presença de falsos positivos. Porém, acreditamos que o treino do avaliador e a utilização do Manual Estruturado para Entrevista HAM$\mathrm{D}^{8}$ (que padronizou as indagações) protegeram o estudo desse tipo de viés.

Dentro da mesma temática do estudo anterior, nosso grupo atualmente desenvolve um novo trabalho (estudo de coorte) que avalia, entre outras variáveis, a depressão na gestação de alto-risco e no pós-parto. Nessa amostra estão incluídas, de acordo com os critérios de classificação para gestação de alto risco do Ministério da Saúde, todas as mães adolescentes atendidas nos ambulatórios de referência para atendimento da gestação de alto risco da cidade de Pelotas no período de julho a outubro de 2006.

Neste estudo utilizamos as escalas EPDS,${ }^{6}$ Hospital Anxiety and Depression Scale ${ }^{2}$ (HAD) e um modelo de entrevista clínica proposto no Mini International Neuropsychiatric Interview ${ }^{1}$ (MINI). Esta metodologia nos permitirá aferir com melhor precisão os quadros depressivos, as co-morbidades e a história psiquiátrica, além de fornecer uma comparação dos resultados antes e depois do parto. A simples escolha do EPDS ou PDSS poderia minimizar a possível dúvida teórica sobre a utilização do HAM-D. Entretanto, não dirimiria a dúvida a respeito do diagnóstico e de eventuais co-morbidades presentes.

De qualquer maneira, gostaríamos mais uma vez de agradecer a contribuição trazida ao nosso estudo pela questão levantada pelo grupo de colegas da Universidade de Pernambuco, assim como lhes dar nossos parabéns pelo trabalho com puérperas adolescentes, um tema onde toda produção realizada é de extrema relevância.

Inácia Gomes da Silva Moraes Augusto Duarte Faria Escola de Psicologia Universidade Católica de Pelotas. Pelotas, RS Ricardo Tavares Pinheiro Ricardo Azevedo da Silva

Paulo Luis Rosa Sousa Programa de Pós-Graduação em Saúde e Comportamento Universidade Católica de Pelotas. Pelotas, RS Bernardo Lessa Horta Programa de Pós-Graduação em Epidemiologia Universidade Federal de Pelotas. Pelotas, RS

\section{REFERÊNCIAS}

1. Amorim P. Mini International Neuropsychiatric Interview (MINI): validation of a short structured diagnostic psychiatric interview. Rev Bras Psiquiatr. 2000;22:106-15.

2. Botega NJ, Bio MC, Zomignani MA, Garcia Jr, Pereira WA. Transtornos do humor em enfermaria de clínica médica e validação de escala de medida (HAD) de ansiedade e depressão. Rev Saúde Pública. 1995;29:355-63.

3. Cantilino A. Tradução para o português e estudo de validação da Postpartum Depression Scale na população brasileira [dissertação de mestrado]. Recife: Universidade Federal de Pernambuco; 2003.

4. Moraes IGS, Pinheiro RT, Silva RA, Horta BL, Sousa PLR, Faria AD. Prevalência da depressão pós-parto e fatores associados. Rev Saúde Pública. 2006;40:65-70.
5. Moreno RA, Moreno DH. Escalas de avaliação clínica em psiquiatria e psicofarmacologia: escalas de avaliação para depressão de Hamilton (HAM-D) e Montgomery-Asberg (MADRS). Rev Psiquiatr Clín. 1998;25(5):1-17.

6. Santos MF, Martins FC, Pasquali L. Escala de autoregistro de depressão pós-parto: estudo no Brasil. In: Gorenstein C, Andrade LH, Zuardi AW, editores. Escalas de avaliação clínica em psiquiatria e psicofarmacologia. São Paulo: Lemos Editorial; 2000. p. 97-101.

7. Snaith P. What do depression rating scales measure? Br J Psychiatry. 1993;163:283-98.

8. Williams JB. A structured interview guide for the Hamilton Depression Rating Scale. Arch Gen Psychiatry.1988;45:742-3. 